\section{RECEPTOR PHYSIOLOGY}

\section{It's the NMDA receptor, but not as we know it}

\begin{abstract}
Unlike most NMDA ( $N$-methylD-aspartate) receptor (NMDAR) subunit combinations, which require glycine and glutamate for activation, NMDARs that contain both NR1 and NR3 subunits (NR1/NR3 receptors) are activated by glycine alone. This property and other unusual properties of this receptor subtype were discovered following experiments in recombinant expression systems but had been difficult to demonstrate in vivo. A paper resulting from an international collaboration between the laboratories of Stys, Lipton and Heinemann now demonstrates the presence of native NR1/NR3 receptors in the myelin surrounding the optic nerve and show that the particular properties of this receptor are ideally suited to its function in the unique environment of the myelin sheath.
\end{abstract}

The study of native NMDARs has typically been performed in brain slices. However, this preparation cannot be used to study NR1/ NR3 receptors because they rapidly desensitize in the presence of glycine, and brain slices contain large amounts of this transmitter owing to the way in which they are prepared. To avoid this problem, Lipton and colleagues devised a less invasive technique involving largely intact optic nerves from mice and rats. They used a $\mathrm{Ca}^{2+}$-sensitive dye as an indicator of receptor channel opening, NR3A knockout mice and selective pharmacological agents to dissect the activation criteria for this receptor subtype.

The authors found that NR1/NR3 receptors were present in the myelinic portions of the nerve but not in the oligodendrocyte soma. The receptors were activated by $\mathrm{D}$-serine, an agonist of NMDAR glycinebinding sites. This effect was blocked by the NMDAR glycine binding site antagonist CNQX, and it was absent in mice lacking the NR3A subunit. As NR2 subunits are also present in myelin, the authors administered D-aminophosphonovalerate (DAP5), a specific antagonist at glutamate binding sites on NR2 subunits, and found that this had no effect on the response to $\mathrm{D}$-serine. This supports the notion that the NR1/NR3 receptors can be activated by glycine alone and that they generate $\mathrm{Ca}^{2+}$ rises independently of NR2-containing receptors.

What is the function of NR1/NR3 receptors in the myelinic compartment and why are they localized exclusively in myelin and not the oligodendrocyte soma? The myelinic compartment has only minute volumes of cytoplasm, such that small changes in ion concentration acting over nanometre distances can exert substantial effects. The authors propose that the intrinsic properties of the NR1/NR3 receptors - specifically, a lower $\mathrm{Ca}^{2+}$ permeability and a higher resistance to $\mathrm{Mg}^{2+}$ blockade than other NMDA receptors - make them ideally suited to prevent $\mathrm{Ca}^{2+}$ overload in this environment. Furthermore, the research has implications for white matter disorders that are associated with neurotoxicity, such as multiple sclerosis.

Sian Lewis

\section{ORIGINAL RESEARCH PAPER}

Piña-Crespo, J. C. et al. Excitatory glycine responses of CNS myelin mediated by NR1/NR3 "NMDA" receptor subunits. J. Neurosci. 30, 11501-11505 (2010)

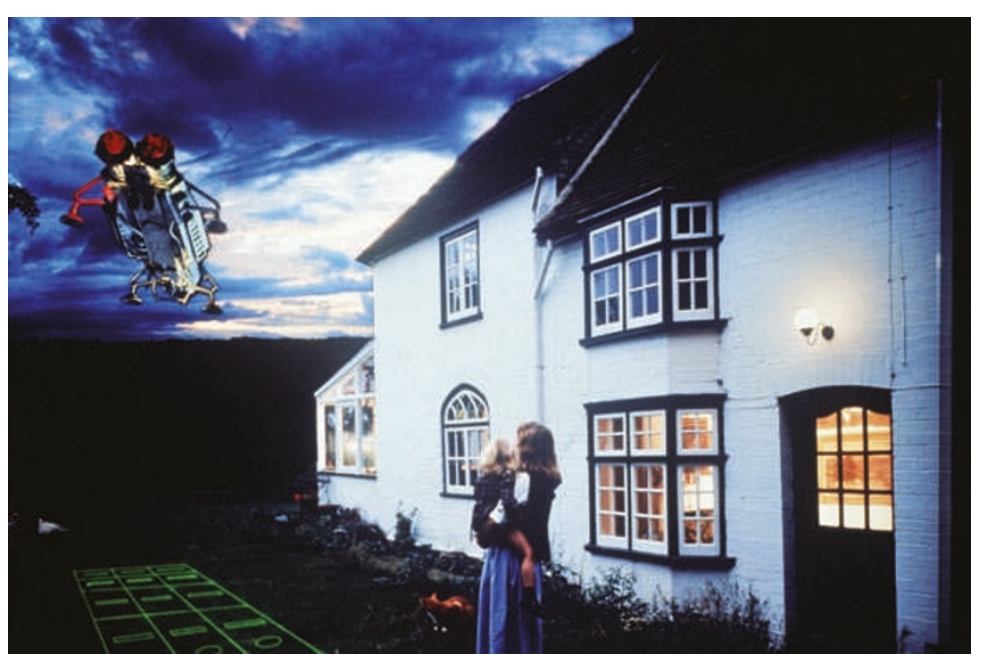

